\title{
Temporal whole field sawtooth flicker without a spatial component elicits a myopic shift following optical defocus irrespective of waveform direction in chicks
}

\author{
Melanie J Murphy ${ }^{\text {Corresp., }}{ }^{1}$, Nina Riddell ${ }^{1}$, David P Crewther ${ }^{2}$, David Simpson ${ }^{3}$, Sheila G Crewther ${ }^{1}$ \\ ${ }^{1}$ School of Psychology \& Public Health, La Trobe University, Melbourne, Victoria, Australia \\ 2 Centre for Human Psychopharmacology, Swinburne University of Technology, Melbourne, Victoria, Australia \\ 3 Brain Sciences Institute, Swinburne University of Technology, Melbourne, Victoria, Australia \\ Corresponding Author: Melanie J Murphy \\ Email address: m.murphy@latrobe.edu.au
}

Purpose. Myopia (short-sightedness) is the commonest visual disorder and greatest risk factor for sight threatening secondary pathologies. Myopia and hyperopia can be induced in animal models by rearing with optical lens defocus of opposite sign. The degree of refractive compensation to lens-induced defocus in chicks has been shown to be modified by directionally drifting sawtooth spatio-temporal luminance diamond plaids, with Fast-ON sawtooth spatio-temporal luminance profiles inhibiting the myopic shift in response to negative lenses, and Fast-OFF profiles inhibiting the hyperopic shift in response to positive lenses. What is unknown is whether similar sign-of-defocus dependent results produced by spatiotemporal modulation of sawtooth patterns could be achieved by rearing chicks under whole field low temporal frequency sawtooth luminance profiles at 1 or $4 \mathrm{~Hz}$ without a spatial component, or whether such stimuli would indiscriminately elicit a myopic shift such as that previously shown with symmetrical (or near-symmetrical) low frequency flicker across a range of species. Methods. Hatchling chicks ( $n=$ 166) were reared from days five to nine under one of three defocus conditions (No Lens, +10D lens, or $10 \mathrm{D}$ lens) and five light conditions (No Flicker, $1 \mathrm{~Hz}$ Fast-ON/Slow-OFF sawtooth flicker, 4Hz Fast-ON/SlowOFF sawtooth flicker, $1 \mathrm{~Hz}$ Fast-OFF/Slow-ON sawtooth flicker, or 4Hz Fast-OFF/Slow-ON sawtooth flicker). The sawtooth flicker was produced by light emitting diodes (white LEDs, 1.2 - 183 Lux), and had no measurable dark phase. Biometrics (refraction and ocular axial dimensions) were measured on day nine. Results. Both $1 \mathrm{~Hz}$ and $4 \mathrm{~Hz}$ Fast-ON and Fast-OFF sawtooth flicker induced an increase in vitreous chamber depth that was greater in the presence of negative compared to positive lens defocus. Both sawtooth profiles at both temporal frequencies inhibited the hyperopic shift in response to +10D lenses, whilst full myopic compensation (or over-compensation) in response to -10D lenses was observed.

Conclusions. Whole field low temporal frequency Fast-ON and Fast-OFF sawtooth flicker induces a generalized myopic shift, similar to that previously shown for symmetrical sine-wave and square-wave flicker. Our findings highlight that temporal modulation of retinal ON/OFF pathways per se (without a spatial component) is insufficient to produce strong sign-of-defocus dependent effect. 
1

2 Temporal Whole Field Sawtooth Flicker without a Spatial Component Elicits a Myopic

3 Shift Following Optical Defocus Irrespective of Waveform Direction in Chicks

4

5 Melanie J. Murphy ${ }^{1}$, Nina Riddell ${ }^{1}$, David P. Crewther ${ }^{2}$, David Simpson ${ }^{3}$ and Sheila G.

6 Crewther ${ }^{1}$

7

8 1. School of Psychology and Public Health, La Trobe University, Melbourne, Victoria Australia.

9 2. Centre for Human Psychopharmacology, Swinburne University of Technology, Hawthorn,

10 Melbourne, Victoria, Australia.

11 3. Brain Sciences Institute, Swinburne University of Technology, Hawthorn, Melbourne,

12 Victoria, Australia.

13

14 Corresponding Author

15 Melanie Murphy

16 E: M.Murphy@1atrobe.edu.au 


\begin{abstract}
Purpose. Myopia (short-sightedness) is the commonest visual disorder and greatest risk factor for sight threatening secondary pathologies. Myopia and hyperopia can be induced in animal models by rearing with optical lens defocus of opposite sign. The degree of refractive compensation to lens-induced defocus in chicks has been shown to be modified by directionally drifting sawtooth spatio-temporal luminance diamond plaids, with Fast-ON sawtooth spatiotemporal luminance profiles inhibiting the myopic shift in response to negative lenses, and FastOFF profiles inhibiting the hyperopic shift in response to positive lenses. What is unknown is whether similar sign-of-defocus dependent results produced by spatio-temporal modulation of sawtooth patterns could be achieved by rearing chicks under whole field low temporal frequency sawtooth luminance profiles at 1 or $4 \mathrm{~Hz}$ without a spatial component, or whether such stimuli would indiscriminately elicit a myopic shift such as that previously shown with symmetrical (or near-symmetrical) low frequency flicker across a range of species.
\end{abstract}

Methods. Hatchling chicks $(n=166)$ were reared from days five to nine under one of three defocus conditions (No Lens, +10D lens, or -10D lens) and five light conditions (No Flicker, 1Hz Fast-ON/Slow-OFF sawtooth flicker, 4Hz Fast-ON/Slow-OFF sawtooth flicker, 1Hz FastOFF/Slow-ON sawtooth flicker, or $4 \mathrm{~Hz}$ Fast-OFF/Slow-ON sawtooth flicker). The sawtooth flicker was produced by light emitting diodes (white LEDs, 1.2 - 183 Lux), and had no measurable dark phase. Biometrics (refraction and ocular axial dimensions) were measured on day nine.

Results. Both $1 \mathrm{~Hz}$ and $4 \mathrm{~Hz}$ Fast-ON and Fast-OFF sawtooth flicker induced an increase in vitreous chamber depth that was greater in the presence of negative compared to positive lens defocus. Both sawtooth profiles at both temporal frequencies inhibited the hyperopic shift in response to $+10 \mathrm{D}$ lenses, whilst full myopic compensation (or over-compensation) in response to $-10 \mathrm{D}$ lenses was observed.

Conclusions. Whole field low temporal frequency Fast-ON and Fast-OFF sawtooth flicker induces a generalized myopic shift, similar to that previously shown for symmetrical sine-wave and square-wave flicker. Our findings highlight that temporal modulation of retinal ON/OFF pathways per se (without a spatial component) is insufficient to produce strong sign-of-defocus dependent effect. 
47

\section{Introduction}

Most species are born with hyperopic eyes that, with normal visual experience, increase in size during early development to achieve emmetropia (i.e., no refractive error) (Norton \& Siegwart Jr 1995; Rabin et al. 1981; Stone et al. 1995; Troilo \& Wallman 1991; Wildsoet 1997). This emmetropization process is controlled by complex gene-environment interactions (Fan et al. 2014; Morgan \& Rose 2005; Verhoeven et al. 2013). Epidemiological studies have demonstrated that exposure to environmental risk factors (such as spending limited time outdoors (Rose et al. 2008) or doing prolonged near-work (Huang et al. 2015)) can predispose the eye to excessive growth resulting in myopia (short-sightedness). Myopia is now the most common ocular disorder (Schneider et al. 2010) with rapidly increasing prevalence and severity worldwide (Lee et al. 2013; Seet et al. 2001), making the development of treatments to control ocular growth a health and socioeconomic priority.

Ocular growth has been extensively studied in animals, where myopia can be induced by rearing with negatively powered defocusing lenses affixed over the eye or form deprivation via lid suture or plastic occluders (Hodos \& Kuenzel 1984; Irving et al. 1992; Schaeffel et al. 1988; Wallman et al. 1978; Wiesel \& Raviola 1977), and hyperopia (long-sightedness) can be induced by positive lenses (Irving et al. 1992; Schaeffel et al. 1988). Ocular growth and refractive shifts in these animal models can also be mediated by pharmacological manipulations of retinal ON/OFF pathway activity (Crewther \& Crewther 1990; Crewther et al. 1996; Ehrlich et al. 1990; Smith et al. 1991). Suppression of ON-pathway activity by L- $\alpha$-aminoadipic acid in chicks and kittens decreases axial growth in normally developing animals (Crewther \& Crewther 1990; Crewther et al. 1996; Crewther \& Crewther 2003). Conversely, OFF-pathway suppression by D- $\alpha-$ aminoadipic acid increases axial growth in normally developing chicks (Crewther \& Crewther 1990), and inhibits optically-induced growth changes (Crewther \& Crewther 1990; Crewther et al. 1996; Crewther \& Crewther 2003). More recent gene knockout studies have demonstrated an increased susceptibility to FD in mice with ON-pathway defects (Chakraborty et al. 2015; Pardue et al. 2008), consistent with the effects of ON-pathway suppression in the chick FD model (Crewther \& Crewther 1990). Mice with an OFF-pathway defect did not develop myopia in response to FD (although this latter result may reflect the genetic background) (Chakraborty et al. 2014). 
Although pharmacological and genetic studies have provided strong evidence implicating retinal

80 effects of visual stimuli designed to preferentially activate $\mathrm{ON}$ - or OFF-systems. One of the first studies was a notable paper by Schwahn \& Schaeffel 1997, (Schwahn \& Schaeffel 1997) who raised chicks in flickering light of different temporal frequencies (12 and $6 \mathrm{~Hz}$ ) and different duty cycles (4-75\% ratio of light to dark periods) produced by rotating chopper disks. Not surprisingly, it was found that the extent to which FD and defocus-induced myopia could be suppressed was related to the length of the duty cycle, suggesting that myopia is related to dark duration and OFF pathway activation. Interestingly, there was no observable suppression of hyperopia.

In 2002, Crewther and Crewther (Crewther \& Crewther 2002) reared chicks in an environment lit with directionally moving sawtooth diamond plaids based on the human Brücke-Bartley Effect whereby a moving Fast-ON/Slow-OFF plaid has a perceptual darkening effect, and a FastOFF/Slow-ON spatiotemporal luminance profile creates a brightening effect (Cavanagh \& Anstis 2018; Cavanagh \& Anstis 1986). Chicks reared with Fast-ON sawtooth plaid illumination compensated fully to positive, but not to negative lenses. Conversely, chicks reared with FastOFF sawtooth plaid illumination compensated fully to negative, but not positive, lenses (Crewther \& Crewther 2002). More recently, Riddell et al (Riddell et al. 2016) examined the electrophysiological basis of the Brücke-Bartley Effect in toad eye cups recordings and showed a sustained direct-current (DC) trans-tissue potential to drifting gratings. As in the human experience of moving sawtooth gratings, the sustained DC potential effect was greater for FastOFF (brightening effect) compared to Fast-ON sawtooth (darkening effect), suggesting that the asymmetries in DC potential originate in the photoreceptoral response to Fast-ON and FastOFFprofiles. Thus, the ability of this paradigm to inhibit growth in a sign-of-defocus dependent manner that allows for potential localisation of structural origins, suggests that environmental modulation of the spatio-temporal activity of the ON/OFF pathways could represent a promising non-invasive treatment avenue for refractive errors using therapies aimed at the outer retina, as suggested by Crewther (Crewther 2000). 
109 What remains to be seen is whether the same inhibitory effects on refractive compensation

110 produced by spatio-temporal modulation of sawtooth patterns, can be achieved using whole field,

111 low temporal frequency sawtooth luminance profiles at 1 or $4 \mathrm{~Hz}$ without a spatial component, or

112 whether such stimuli would indiscriminately elicit a myopic shift such as that previously shown

113 to result from symmetrical (or near-symmetrical) low frequency flicker across a range of species

114 (Britton et al. 2014; Cheng et al. 2004; Cremieux et al. 1989; Crewther et al. 2006; Di et al.

115 2013a; Di et al. 2013b; Di et al. 2014; Li et al. 2016; Luo et al. 2017; Pan et al. 2017; Wang et al.

116 2013; Yu et al. 2011; Zhi et al. 2013).

117 In an attempt to resolve this question, Crewther et al. (Crewther et al. 2006) investigated the

118 effect of whole field, low frequency flicker and found a myopic shift for all lens conditions with

119 a stimulus comprised of an asymmetric light pulse similar to that of a Slow ON/Fast OFF

120 sawtooth and 1:2 ratio light:dark cycle. However, due to the extended dark period in the stimulus

121 profile created by the use of a halogen globe, the stimulus utilized in this study failed to replicate

122 a true temporal illuminance-based Brücke-Bartley effect with a reversible sawtooth profile as

123 produced in the 2002 study by the same lab, preventing an examination of the effect of

124 continuous full field light dark transitions on directional growth following suppression of either

125 the ON or OFF pathways.

126

127 Thus, the present study aimed to re-investigate the effects of whole field low frequency Fast-ON

128 and Fast-OFF sawtooth flicker on refractive compensation to defocusing lenses in chick using

129 the white light emitting diodes (LEDs) LEDS. LEDS are a more precise system for controlling

130 temporal luminance modulation and able to deliver reversible asymmetric light profiles (Fast-

$131 \mathrm{ON} / \mathrm{Slow}-\mathrm{OFF}$ and Fast-OFF/Slow-ON) that closely mimicked the temporal aspects of the

132 spatiotemporal sawtooth plaid paradigm (Crewther \& Crewther 2002).

133

134 Materials \& Methods

135 Animals and Rearing

136 A total of 166 male hatchling chicks (Leghorn/New Hampshire) were obtained from a local

137 hatchery and raised from day five to nine in a light (12/12 hour day/night cycle) and temperature

$138\left(30 \pm 0.5^{\circ} \mathrm{C}\right)$ controlled enclosure (height: $0.5 \mathrm{~m}$, length: $1.0 \mathrm{~m}$, width: $\left.0.75 \mathrm{~m}\right)$. On day five, 
139 chicks were fitted with either a monocular +10D or -10D lens, or left as No Lens controls. These

140 defocusing goggles were made from modified human PMMA contact lenses $(8.1 \mathrm{~mm}$ in

141 diameter) attached to Velcro $\subset$ and affixed to the periocular feathers of the right eye. Within

142 each lens condition, chicks were randomly assigned to one of five light profiles: normal light

143 (referred to herein as No Flicker [NF]), 1Hz Fast-ON/Slow-OFF flicker, 4Hz Fast-ON/Slow-

144 OFF flicker, $1 \mathrm{~Hz}$ Fast-OFF/Slow-ON flicker, or 4Hz Fast-OFF/Slow-ON flicker. All light

145 profiles followed a 12/12 hour day/night cycle.

146

147

The five light profiles were delivered using LEDs mounted to the roof of the enclosure. Ambient

light was maintained constantly at 183 lux during the 12 hour day cycle (No Flicker) or

modulated (using the same lamp) at frequencies of either $1 \mathrm{~Hz}$ or $4 \mathrm{~Hz}$ where light levels varied

150 between a minimum of 1.2 lux to a maximum of 183 lux, with an average light level of 97 lux. Ambient lighting in the No Flicker condition was maintained at the maximum (rather than the mean) of the flicker conditions to facilitate comparison with our previous study with a 'no flicker' condition of 180 lux (Crewther et al. 2006). For the flicker conditions, the LEDs produced a whole field sawtooth temporal luminance profile with a strict linear rise or fall in luminance achieved through feedback control circuitry connected to a DC power supply with computer input to implement waveform and frequency (for specifications please refer to material, ensuring that there is absolutely no change in the spectral distribution with brightness. Figure 1 shows the sawtooth wave profile recorded using a PowerLab (AD Instruments, Melbourne, Australia) via a light probe situated in the animal enclosure.

\section{Biometric Analysis}

On day nine, chicks were anaesthetized (Ketamine 45mg/kg: Xylazine 4.5mg/kg i.m.) and both eyes were refracted by retinoscopy (Keeler, Vista Diagnostic Instruments) and axial dimensions were obtained from the average of at least three A-Scan ultrasonography traces (A-Scan III, TSL: Teknar, Inc. St Louis, $7 \mathrm{MHz}$ probe). Each trace from the A-Scan provided peaks 
170 retina), the distance from the posterior pole of the lens to the inner limiting membrane of the

171 retina and the depth of the vitreous chamber (all measured in millimeters); subtracting the lens to

172 retina measurement from the axial length gave a measure of the depth central to the axis of the

173 anterior chamber. All procedures were conducted in strict accordance with La Trobe University

174 Animal Ethics Committee guidelines (Approval Number 08/30) and adhered to the NHMRC of

175 Australia and ARVO Statements for the use of Animals in Ophthalmic and Vision Research.

176

177 Data Analysis

178 To control for differences in eye size within groups, refractive and biometric measures were 179 converted to raw score differences between experimental and fellow eyes. To assess whether 180 changes in ocular size were due to the experimental manipulation a 3 (lens condition $\times$ frequency $181 \times$ light profile) way ANOVA was utilized. Student Newman Keuls or Games-Howell post hoc 182 tests were performed as appropriate.

\section{Results}

185

186

187

188

189

190

191

192

193

194

195

196

197

198

199

200

Table 1 reports the raw scores for refraction and axial length (mean and standard error of the mean) for each experimental group.

\section{[Table 1]}

Figure 2 illustrates the mean differences (Experimental - Fellow Eye) in biometric measures across groups.

[Figure 2]

As can be seen in Figure 2a, all chicks wearing -10D lenses developed myopia under all light conditions. Hyperopic refractive compensation was also seen in eyes reared with $+10 \mathrm{D}$ lenses under all flicker conditions. Fast OFF flicker at a frequency of $1 \mathrm{~Hz}$ produced the most notable impact on refraction, with eyes reared with -10D lenses under this condition displaying approximately $2 \mathrm{D}$ more myopia than other $-10 \mathrm{D}$ lens groups. Fast OFF flicker also resulted in the greatest suppression of hyperopia in eyes wearing +10D lenses. Chicks reared without a lens 
201 were largely plano under all light conditions. Simple main effects analysis revealed a significant 202 effect of light profile $\left(\mathrm{F}_{1,149}=10.62, \mathrm{p}=.0001\right)$, lens $\left(\mathrm{F}_{2,149}=453.65, \mathrm{p}<.0001\right)$ and frequency $203\left(\mathrm{~F}_{2,149}=13.32, \mathrm{p}<.0001\right)$. Significant interactions were also observed between lens and light

$204\left(\mathrm{~F}_{2,149}=3.89, \mathrm{p}=.023\right)$, lens and frequency $\left(\mathrm{F}_{2,149}=3.98, \mathrm{p}=.021\right)$, light and frequency $\left(\mathrm{F}_{1,149}\right.$ $205=13.17, \mathrm{p}=.0001)$, and lens and light and frequency $\left(\mathrm{F}_{2,149}=3.28, \mathrm{p}=.04\right)$.

206

207

208

209

210

211

212

213

214

215

216

217

218

219

220

221

222

223

224

225

226

227

228

229

230

231

Post hoc analysis comparing the effect of flicker frequency on refractive state revealed that eyes reared with -10D lenses were significantly more myopic under Fast OFF light conditions with 1 $\mathrm{Hz}$ flicker, in comparison to $4 \mathrm{~Hz}$ and no flicker (NF) conditions (see Table 2). No difference in refraction was found between flicker frequencies with a Fast ON sawtooth profile for eyes reared with -10D lenses. Under Fast OFF sawtooth conditions, chicks wearing +10D lenses were significantly less hyperopic when raised with $1 \mathrm{~Hz}$ flicker compared to $4 \mathrm{~Hz}$ and no flicker conditions (see Figure 2a). Chicks reared under Fast OFF light were significantly less hyperopia than all other chicks wearing +10 D lenses. Refractions were significantly different between all lens groups when compared within frequency and sawtooth conditions. Comparisons between the effect of the sawtooth profile on refraction revealed that Fast OFF with $1 \mathrm{~Hz}$ flicker resulted in significantly greater myopia than Fast ON $+1 \mathrm{~Hz}$ or NF light conditions with $-10 \mathrm{D}$ lenses, and significantly less hyperopia with $+10 \mathrm{D}$ lens-wear. A summary of post hoc analyses is presented in Supplementary Table 1.

An overall increase in axial length in response to -10D lenses was observed, with eyes reared under Fast OFF flicker at $1 \mathrm{~Hz}$ showing the longest eyes (Figure 2b). Fast OFF flicker at $1 \mathrm{~Hz}$ also resulted in longer axial length with $+10 \mathrm{D}$ lens-wear in comparison to NF control, as did Fast ON flicker at $1 \mathrm{~Hz}$. No Lens chicks showed slight increases in length for all conditions. As expected, all other $+10 \mathrm{D}$ lens groups showed an overall decrease in axial length compared to No Lens groups. Simple main effects analysis revealed a significant effect of lens $\left(F_{2,149}=131.00\right.$, $\mathrm{p}<.0001)$ and frequency $\left(\mathrm{F}_{2,149}=13.53, \mathrm{p}<.0001\right)$. A significant interaction effect was obtained for lens and frequency $\left(\mathrm{F}_{2,149}=4.46, \mathrm{p}=.013\right)$. Post hoc analysis confirmed the observation that $1 \mathrm{~Hz}$ flicker for both sawtooth profiles led to longer axial lengths for $+10 \mathrm{D}$ lens 
232 conditions.

233

234 Consistent with axial length measures, Figure 2c shows an overall increase in vitreous chamber

235 depth in response to -10D lenses for all light conditions, and a notable decrease in vitreous

236 chamber depth in response to +10D lenses for all light conditions except for Fast OFF and Fast

237 ON $1 \mathrm{~Hz}$ flicker in comparison to No Lens groups. No Lens chicks showed a slight increase in

238 vitreous chamber depth. Simple main effects analysis revealed a significant effect of lens $\left(\mathrm{F}_{2,149}\right.$

$239=158.01, \mathrm{p}<.0001)$ and frequency $\left(\mathrm{F}_{2,149}=13.37, \mathrm{p}<.0001\right)$, and a significant interaction

240 between lens and frequency $\left(\mathrm{F}_{2,149}=4.47, \mathrm{p}=.013\right)$. Post hoc analysis again confirmed the

241 observation that $1 \mathrm{~Hz}$ flicker for both sawtooth profiles led to longer vitreous chamber depths for

$242+10 \mathrm{D}$ lens conditions.

243

244

As expected, a great degree of variability was observed in anterior chamber depth in response to lens wear (Figure $2 \mathrm{~d})$. A significant interaction between lens and frequency $\left(\mathrm{F}_{2,149}=5.88, \mathrm{p}=\right.$ .004) was found. Post hoc tests showed that $4 \mathrm{~Hz}$ flicker for both Fast OFF and Fast ON profiles led to shallower anterior chamber depths for $+10 \mathrm{D}$ lens conditions.

\section{Discussion}

Previous studies have shown that low frequency sine-wave and square-wave flicker induces a generalized myopic shift in a range of species (Britton et al. 2014; Cheng et al. 2004; Cremieux et al. 1989; Di et al. 2013a; Di et al. 2013b; Di et al. 2014; Li et al. 2016; Luo et al. 2017; Yu et al. 2011; Zhi et al. 2013). In the present study, we demonstrate that whole field temporal Fast ON and Fast OFF sawtooth flicker with no spatial component or dark phase induces a general increase in vitreous chamber depth and a subsequent myopic shift that is greater in the presence of defocus (particularly positive defocus), and greater at lower temporal frequencies (i.e., $1 \mathrm{~Hz}$ versus $4 \mathrm{~Hz}$ ). The largest effects occurred for $1 \mathrm{~Hz}$ Fast OFF flicker, which strongly inhibited refractive compensation to positive lenses and induced over-compensation to negative lenses. Although this latter finding is in agreement with our previous study of spatiotemporal sawtooth flicker (Crewther \& Crewther 2002), on the whole, these results suggest that whole field temporal modulation of retinal ON/OFF pathways does not produce the strong sign-of-defocus 
262 dependent effects previously demonstrated for spatiotemporal ON/OFF stimuli (Crewther \&

263 Crewther 2002).

264

265

266

267

268

269

270

271

272

273

274

275

276

277

278

279

280

281

282

283

284

285

286

287

288

289

290

291

As light levels can affect responses to lens and occluder wear (Norton \& Siegwart 2013), further research is needed to rule out a contribution from differing mean illuminance between the temporal sawtooth in the present study and the spatiotemporal sawtooth in our previous study (97 and 387 lux, respectively), and between the no flicker and flicker conditions in the present study (183 and 97 lux, respectively). Past research has shown that chicks reared under very dim lighting ( $\leq 50$ lux) for weeks to months develop longer axial lengths and a more myopic refraction (Bercovitz et al. 1972; Cohen et al. 2011; Cohen et al. 2012; Lauber \& Oishi 1987). However, shorter duration studies have shown no difference in refractive development following 4 days of occluder wear under 50 lux versus 500 lux lighting (Ashby et al. 2009), or following 7 days of wearing neutral density filters (0.5-1 log unit attenuation) versus clear filters under 500 lux lighting (Feldkaemper et al. 1999). These latter findings suggest that the contribution of mean illuminance to refractive outcomes across conditions is likely to be minimal. That said, a comparison of the present findings with those of our past study investigating near-symmetrical flicker (Crewther et al. 2006) is not inconsistent with a contribution from mean luminance in driving refractive change. This past study investigated the effects of flicker with a gradual rise and slightly sharper decline (approximating a Fast-OFF profile), and variable duration dark phase (longest for $1 \mathrm{~Hz}$ flicker and shortest for $4 \mathrm{~Hz}$ flicker) (Crewther et al. 2006). In this previous study, the $1 \mathrm{~Hz}$ flicker condition (which had the lowest mean luminance) caused the strongest inhibition of compensation to positive lenses, and all frequencies caused a similar degree of over-compensation to negative lenses. The refractive shifts observed in the present study, where there was no measurable dark phase and thus a slightly higher mean luminance, were similar but less extreme. The results of these two studies are also interesting in the context of previous research showing a correlation between the degree of myopia suppression by flicker and the duration of the dark phase at high frequencies (with longer dark phases conferring greater suppression) (Schwahn \& Schaeffel 1997). Our previous study (Crewther et al. 2006) demonstrated equivalent over-compensation to negative lenses despite large differences in the duration of the dark phase at 1,2 , and $4 \mathrm{~Hz}$. These results demonstrate that the correlation 
292 between the flicker dark phase and the degree of myopia induction does not hold at low temporal

293 frequencies when the mean illuminance is not controlled.

294

295

Previous physiological research in monkey (Khan et al. 2005) and toad eye cup (Riddell et al.

296

297

298

299

300

301

302

303

304

305

306

307

308

309

310

311

312

313

314

315

316

317

318

319

320

321

322 2016) indicates that temporal and spatiotemporal sawtooth modulation produces complex postreceptoral ON/OFF pathway asymmetries, with push-pull interactions evident between ON- and OFF- bipolar cell contributions to Fast ON and Fast OFF waveforms in both paradigms (Khan et al. 2005; Riddell et al. 2016). Although both paradigms mediate ON/OFF pathway activity in a polarity-specific manner, their effects on gross trans-retinal potentials are vastly different. Spatiotemporal sawtooth produces a sustained trans-retinal potential increase (presumably resulting from the summation of potentials from different local retinal regions), that has asymmetries at the photoreceptor-RPE level as well as post-receptorally (Riddell et al. 2016). The important difference between the Crewther and Crewther's 2002 (Crewther \& Crewther 2002) stimuli and stimuli utilised in the current study is the removal of the spatial aspects of each local diamond stimulus. The current stimulation procedure is a full field sawtooth $\mathrm{ON}$ to OFF, or OFF to ON temporal modulation of the environment. The neural response to whole field temporally modulated light is an 'entire retina' photoreceptor response that results in a global change in retinal transmembrane potential (Khan et al. 2005). In comparison, the neural response to the spatial plaid components of the diamond pattern is expected to allow relatively localized spatial and temporal buffering of the change of potential across the retina (Riddell et al. 2016). Such differences in their effects can be conceptualized in terms of spatial buffering of ions. Each change in illuminance, whether temporal only or spatiotemporal, results in a large efflux of sodium ions into the outer segments and potassium out in the subretinal space during darkness and reversal of this process during light onset (See (Bialek \& Miller 1994; Gallemore et al. 1997; Hamann 2002; Li et al. 1994; Steinberg 1985; Steinberg et al. 1985). It is proposed that the reduced effect $4 \mathrm{~Hz}$ flicker on eye growth is related to the refractory period required to modulate directional change in the buffering of ion distributions in conjunction with the magnitude of the change in transretinal potential caused by the flicker stimulus. Thus, the full field stimulation of the current 2018 study forces ionic changes in the radial dimension, reducing the processes to a 1-dimensional situation across the retina. In comparison, the 2002 spatiotemporal diamonds could allow buffering in the direction tangential to the retinal layers, for example, in the 
323 subretinal space, or indeed within individual RPE cells to produce a directional growth signal in

324 response to signed defocus. Further investigation is warranted to determine the nature of the

325 refractory period for ionic spatial buffering and the impact of spatiotemporal versus temporal

326 frequency signals in driving signed compensation.

327

328

329

330

331

332

333

334

335

336

337

338

339

340

341

342

343

344

345

346

347

348

349

350

351

\section{Conclusions}

The present study demonstrated that both whole field low temporal frequency fast ON and fast OFF flicker induce a generalized myopic shift in chicks wearing defocusing lenses, but do not affect refractive development in chicks not wearing lenses. These results are highly similar to our previous findings using near-symmetrical flicker approximating a Fast OFF profile (Crewther et al. 2006), as well as being broadly concordant with the myopic shift shown following exposure to symmetrical flicker in chicks (Britton et al. 2014), mice (Yu et al. 2011), guinea pigs (Cheng et al. 2004; Di et al. 2013a; Di et al. 2013b; Di et al. 2014; Li et al. 2016; Luo et al. 2017; Zhi et al. 2013), and cats (Cremieux et al. 1989). Most importantly, this study highlights the fact that temporal luminance modulation per se is not adequate to induce signed directional refractive compensation. Rather, signed modulation of refractive compensation requires a directional spatiotemporal component which likely effects relative adaptive brightness in local regions across the retina.

\section{Acknowledgements}

The authors would like to thank Sarah Kiely for her assistance with animal rearing and initial analysis and manuscript development, and Loretta Giummarra for her assistance with animal rearing. This work was presented in part as a poster at the annual meeting for the Association for Research in Vision and Ophthalmology, ARVO, Fort Lauderdale, FL, USA, May 2010 (Kiely et al. 2010). 
352

353

354

355

356

357

358

359

360

361

362

363

364

365

366

367

368

369

370

371

372

373

374

375

376

377

378

379

380

381

Ashby R, Ohlendorf A, and Schaeffel F. 2009. The effect of ambient illuminance on the development of deprivation myopia in chicks. Invest Ophthalmol Vis Sci 50:5348-5354. 10.1167/iovs.09-3419

Bercovitz AB, Harrison PC, and Leary GA. 1972. Light induced alterations in growth pattern of the avian eye. Vision Research 12:1253-1254,IN1255-IN1256,1255-1259. 10.1016/00426989(72)90196-4

Bialek S, and Miller SS. 1994. K+ and Cl-transport mechanisms in bovine pigment epithelium that could modulate subretinal space volume and composition. The Journal of physiology 475:401-417.

Britton S, Hanowsky S, and Rucker FJ. 2014. Blue light protects against temporal frequency dependent refractive changes. Investigative Ophthalmology \& Visual Science 55:ARVO Annual Meeting E-Abstract 3598.

Cavanagh P, and Anstis S. 2018. Diamond patterns: Cumulative Cornsweet effects and motioninduced brightening. $i$-Perception 9:2041669518770690.

Cavanagh P, and Anstis SM. 1986. Brightness shift in drifting ramp gratings isolates a transient mechanism. Vision research 26:899-908.

Chakraborty R, Park H, Aung MH, Tan CC, Sidhu CS, Iuvone PM, and Pardue MT. 2014. Comparison of refractive development and retinal dopamine in OFF pathway mutant and C57BL/6J wild-type mice. Mol Vis 20:1318-1327.

Chakraborty R, Park HN, Hanif AM, Sidhu CS, Iuvone PM, and Pardue MT. 2015. ON pathway mutations increase susceptibility to form-deprivation myopia. Exp Eye Res 137:79-83. 10.1016/j.exer.2015.06.009

Cheng ZY, Li JH, Li R, and Xie YB. 2004. [Effects of flashing light on ocular growth and development of myopia in pigmented guinea pigs]. Zhonghua Yan Ke Za Zhi 40:601-604.

Cohen Y, Belkin M, Yehezkel O, Solomon AS, and Polat U. 2011. Dependency between light intensity and refractive development under light-dark cycles. Experimental eye research 92:40-46.

Cohen Y, Peleg E, Belkin M, Polat U, and Solomon AS. 2012. Ambient illuminance, retinal dopamine release and refractive development in chicks. Exp Eye Res 103:33-40. $10.1016 /$ j.exer.2012.08.004 
382 Cremieux J, Orban GA, Duysens J, Amblard B, and Kennedy H. 1989. Experimental myopia in

383

384

385

386

387

388

389

390

391

392

393

394

395

396

397

398

399

400

401

402

403

404

405

406

407

408

409

410

411 cats reared in stroboscopic illumination. Vision Res 29:1033-1036.

Crewther D, and Crewther S. 1990. Pharmacological modification of eye growth in normally reared and visually deprived chicks. Current eye research 9:733-740.

Crewther D, Crewther S, and Xie R. 1996. Changes in eye growth produced by drugs which affect retinal ON or OFF responses to light. Journal of ocular pharmacology and therapeutics 12:193-208.

Crewther DP. 2000. The role of photoreceptors in the control of refractive state. Progress in retinal and eye research 19:421-457.

Crewther DP, and Crewther SG. 2002. Refractive compensation to optical defocus depends on the temporal profile of luminance modulation of the environment. Neuroreport 13:10291032.

Crewther S, and Crewther DP. 2003. Inhibition of retinal ON/OFF systems differentially affects refractive compensation to defocus. Neuroreport 14:1233-1237.

Crewther SG, Barutchu A, Murphy MJ, and Crewther DP. 2006. Low frequency temporal modulation of light promotes a myopic shift in refractive compensation to all spectacle lenses. Exp Eye Res 83:322-328. 10.1016/j.exer.2005.12.016

Di Y, Liu R, Chu RY, Zhou XT, and Zhou XD. 2013a. Myopia induced by flickering light in guinea pigs: a detailed assessment on susceptibility of different frequencies. Int $J$ Ophthalmol 6:115-119. 10.3980/j.issn.2222-3959.2013.02.01

Di Y, Lu N, Li B, Liu R, Chu RY, Zhou XT, and Zhou XD. 2013b. Effects of chronic exposure to $0.5 \mathrm{~Hz}$ and $5 \mathrm{~Hz}$ flickering illumination on the eye growth of guinea pigs. Curr Eye Res 38:1182-1190. 10.3109/02713683.2013.807931

Di Y, Lu N, Liu R, Chu R, Zhou X, and Zhou X. 2014. The effect of various levels of stroboscopic illumination on the growth of guinea pig eyes. Clinical and Experimental Optometry 97:55-61.

Ehrlich D, Sattayasai J, Zappia J, and Barrington M. 1990. Effects of selective neurotoxins on eye growth in the young chick. Myopia and the control of eye growth:63-88.

Fan Q, Wojciechowski R, Ikram MK, Cheng C-Y, Chen P, Zhou X, Pan C-W, Khor C-C, Tai ES, and Aung T. 2014. Education influences the association between genetic variants and 
412

413

414

415

416

417

418

419

420

421

422

423

424

425

426

427

428

429

430

431

432

433

434

435

436

437

438

439

440

refractive error: a meta-analysis of five Singapore studies. Human molecular genetics 23:546-554.

Feldkaemper M, Diether S, Kleine G, and Schaeffel F. 1999. Interactions of spatial and luminance information in the retina of chickens during myopia development. Exp Eye Res 68:105-115. 10.1006/exer.1998.0590

Gallemore RP, Hughes BA, and Miller SS. 1997. Retinal pigment epithelial transport mechanisms and their contributions to the electroretinogram. Progress in Retinal and Eye Research 16:509-566.

Hamann S. 2002. Molecular mechanisms of water transport in the eye. International review of cytology: Elsevier, 395-431.

Hodos W, and Kuenzel WJ. 1984. Retinal-image degradation produces ocular enlargement in chicks. Invest Ophthalmol Vis Sci 25:652-659.

Huang H-M, Chang DS-T, and Wu P-C. 2015. The association between near work activities and myopia in children - a systematic review and meta-analysis. PloS one 10:e0140419.

Irving EL, Sivak JG, and Callender MG. 1992. Refractive plasticity of the developing chick eye. Ophthalmic Physiol Opt 12:448-456.

Khan NW, Kondo M, Hiriyanna KT, Jamison JA, Bush RA, and Sieving PA. 2005. Primate Retinal Signaling Pathways: Suppressing ON-Pathway Activity in Monkey With Glutamate Analogues Mimics Human CSNB1-NYX Genetic Night Blindness. $J$ Neurophysiol 93:481-492. 10.1152/jn.00365.2004

Kiely S, Kanaris J, Murphy M, Giummarra L, Mortimer A, Crewther D, and Crewther S. 2010. The Effects of Temporal Luminance Modulation on Compensation to Defocus. Investigative Ophthalmology \& Visual Science 51:ARVO Annual Meeting E-Abstract 1734.

Lauber JK, and Oishi T. 1987. Lid suture myopia in chicks. Invest Ophthalmol Vis Sci 28:18511858.

Lee YY, Lo CT, Sheu SJ, and Lin JL. 2013. What Factors are Associated with Myopia in Young Adults? A Survey Study in Taiwan Military Conscripts. Investigative Ophthalmology \& Visual Science 54:1026-1033. DOI 10.1167/iovs.12-10480 
441

442

443

444

445

446

447

448

449

450

451

452

453

454

455

456

457

458

459

460

461

462

463

464

465

466

467

468

469

470

471

Li B, Luo X, Li T, Zheng C, Ji S, Ma Y, Zhang S, and Zhou X. 2016. Effects of Constant Flickering Light on Refractive Status, 5-HT and 5-HT2A Receptor in Guinea Pigs. PLoS One 11:e0167902. 10.1371/journal.pone.0167902

Li J-d, Gallemore RP, Dmitriev A, and Steinberg RH. 1994. Light-dependent hydration of the space surrounding photoreceptors in chick retina. Investigative Ophthalmology \& Visual Science 35:2700-2711.

Luo X, Li B, Li T, Di Y, Zheng C, Ji S, Ma Y, Zhu J, Chen X, and Zhou X. 2017. Myopia induced by flickering light in guinea pig eyes is associated with increased rather than decreased dopamine release. Mol Vis 23:666-679.

Morgan I, and Rose K. 2005. How genetic is school myopia? Progress in retinal and eye research 24:1-38.

Norton T, and Siegwart Jr J. 1995. Animal models of emmetropization: matching axial length to the focal plane. Journal of the American Optometric Association 66:405-414.

Norton TT, and Siegwart JT, Jr. 2013. Light levels, refractive development, and myopia--a speculative review. Exp Eye Res 114:48-57. 10.1016/j.exer.2013.05.004

Pan CW, Wu RK, Liu H, Li J, and Zhong H. 2017. Types of Lamp for Homework and Myopia among Chinese School-Aged Children. Ophthalmic Epidemiol:1-7. $10.1080 / 09286586.2017 .1420204$

Pardue MT, Faulkner AE, Fernandes A, Yin H, Schaeffel F, Williams RW, Pozdeyev N, and Iuvone PM. 2008. High susceptibility to experimental myopia in a mouse model with a retinal on pathway defect. Invest Ophthalmol Vis Sci 49:706-712. 10.1167/iovs.07-0643

Rabin J, Van Sluyters R, and Malach R. 1981. Emmetropization: a vision-dependent phenomenon. Investigative Ophthalmology \& Visual Science 20:561-564.

Riddell N, Hugrass L, Jayasuriya J, Crewther SG, and Crewther DP. 2016. An asymmetric outer retinal response to drifting sawtooth gratings. J Neurophysiol 115:2349-2358. 10.1152/jn.00040.2016

Rose KA, Morgan IG, Ip J, Kifley A, Huynh S, Smith W, and Mitchell P. 2008. Outdoor activity reduces the prevalence of myopia in children. Ophthalmology 115:1279-1285. 10.1016/j.ophtha.2007.12.019

Schaeffel F, Glasser A, and Howland HC. 1988. Accommodation, refractive error and eye growth in chickens. Vision Research 28:639-657. 
472 Schneider J, Leeder SR, Gopinath B, Wang JJ, and Mitchell P. 2010. Frequency, course, and

473

474

475

476

477

478

479

480

481

482

483

484

485

486

487

488

489

490

491

492

493

494

495

496

497

498

499

500

501

502 impact of correctable visual impairment (uncorrected refractive error). Surv Ophthalmol 55:539-560. 10.1016/j.survophthal.2010.02.004

Schwahn HN, and Schaeffel F. 1997. Flicker parameters are different for suppression of myopia and hyperopia. Vision research 37:2661-2673.

Seet B, Wong TY, Tan DT, Saw SM, Balakrishnan V, Lee LK, and Lim AS. 2001. Myopia in Singapore: taking a public health approach. Br J Ophthalmol 85:521-526.

Smith EL, Fox DA, and Duncan GC. 1991. Refractive-error changes in kitten eyes produced by chronic on-channel blockade. Vision research 31:833-844.

Steinberg R. 1985. Interactions between the retinal pigment epithelium and the neural retina. Documenta Ophthalmologica 60:327-346.

Steinberg R, Linsenmeier R, and Griff E. 1985. Retinal pigment epithelial cell contributions to the electroretinogram and electrooculogram. Progress in retinal research 4:33-66.

Stone RA, Lin T, Desai D, and Capehart C. 1995. Photoperiod, early post-natal eye growth, and visual deprivation. Vision research 35:1195-1202.

Troilo D, and Wallman J. 1991. The regulation of eye growth and refractive state: an experimental study of emmetropization. Vision research 31:1237-1250.

Verhoeven VJ, Buitendijk GH, Consortium for Refractive E, Myopia, Rivadeneira F, Uitterlinden AG, Vingerling JR, Hofman A, and Klaver CC. 2013. Education influences the role of genetics in myopia. Eur J Epidemiol 28:973-980. 10.1007/s10654-013-9856-1

Wallman J, Turkel J, and Trachtman J. 1978. Extreme myopia produced by modest change in early visual experience. Science 201:1249-1251.

Wang H, Zhuang K, Gao L, Zhang L, and Yang H. 2013. Increased expression of CCN2 in the red flashing light-induced myopia in guinea pigs. Biomed Res Int 2013:761823. $10.1155 / 2013 / 761823$

Wiesel TN, and Raviola E. 1977. Myopia and eye enlargement after neonatal lid fusion in monkeys. Nature 266:66-68.

Wildsoet C. 1997. Active emmetropization-evidence for its existence and ramifications for clinical practice. Ophthalmic and Physiological Optics 17:279-290.

Yu Y, Chen H, Tuo J, and Zhu Y. 2011. Effects of flickering light on refraction and changes in eye axial length of C57BL/6 mice. Ophthalmic Res 46:80-87. 10.1159/000323179 
503 Zhi Z, Pan M, Xie R, Xiong S, Zhou X, and Qu J. 2013. The effect of temporal and spatial

504 stimuli on the refractive status of guinea pigs following natural emmetropization. Invest $505 \quad$ Ophthalmol Vis Sci 54:890-897. 10.1167/iovs.11-8064

506 


\section{Table $\mathbf{1}$ (on next page)}

Mean ( \pm S.E) refraction and axial length of experimental eyes (EE) and fellow eyes (FE) for each lens, light profile and frequency condition. 


\begin{tabular}{|c|c|c|c|c|c|c|c|c|c|c|c|c|c|c|c|c|}
\hline & & & & $-10 \mathrm{D}$ & & & & & No Le & & & & & $+10 \mathrm{I}$ & & \\
\hline & & $\mathrm{n}$ & $\begin{array}{l}\mathrm{EE} \\
\mathrm{RE} \\
\text { (D) }\end{array}$ & $\begin{array}{l}\mathrm{FE} \\
\mathrm{RE} \\
\text { (D) }\end{array}$ & $\begin{array}{c}\mathrm{EE} \\
\mathrm{AL} \\
(\mathrm{mm})\end{array}$ & $\begin{array}{c}\mathrm{FE} \\
\mathrm{AL} \\
(\mathrm{mm})\end{array}$ & $\mathrm{n}$ & $\begin{array}{l}\text { EE } \\
\text { RE } \\
\text { (D) }\end{array}$ & $\begin{array}{l}\mathrm{FE} \\
\mathrm{RE} \\
\text { (D) }\end{array}$ & $\begin{array}{c}\mathrm{EE} \\
\mathrm{AL} \\
(\mathrm{mm})\end{array}$ & $\begin{array}{c}\mathrm{FE} \\
\mathrm{AL} \\
(\mathrm{mm})\end{array}$ & $\mathrm{n}$ & $\begin{array}{l}\mathrm{EE} \\
\mathrm{RE} \\
\text { (D) }\end{array}$ & $\begin{array}{l}\mathrm{FE} \\
\mathrm{RE} \\
\text { (D) }\end{array}$ & $\begin{array}{c}\mathrm{EE} \\
\mathrm{AL} \\
(\mathrm{mm})\end{array}$ & $\begin{array}{c}\text { FE } \\
\text { AL } \\
(\mathrm{mm} \\
)\end{array}$ \\
\hline \multirow[t]{2}{*}{$\begin{array}{l}\text { Fast } \\
\text { OFF }\end{array}$} & $1 \mathrm{~Hz}$ & 10 & $\begin{array}{c}-12.6 \\
\pm 1.0 \\
4\end{array}$ & $\begin{array}{c}.30 \\
\pm .15\end{array}$ & $\begin{array}{l}9.32 \\
\pm .07\end{array}$ & $\begin{array}{l}8.81 \\
\pm .06\end{array}$ & 9 & $\begin{array}{l}-.56 \\
\pm .47\end{array}$ & $\begin{array}{l}-.14 \\
\pm .28\end{array}$ & $\begin{array}{l}8.81 \\
\pm .06\end{array}$ & $\begin{array}{l}8.75 \\
\pm .05\end{array}$ & 9 & $\begin{array}{c}0.75 \\
\pm 1.1 \\
5\end{array}$ & $\begin{array}{c}.17 \\
\pm .12\end{array}$ & $\begin{array}{l}8.92 \\
\pm .12\end{array}$ & $\begin{array}{l}8.85 \\
\pm .06\end{array}$ \\
\hline & $4 \mathrm{~Hz}$ & 11 & $\begin{array}{c}-7.18 \\
\pm 1.6 \\
8\end{array}$ & $\begin{array}{c}.14 \\
\pm .30\end{array}$ & $\begin{array}{l}9.03 \\
\pm .07\end{array}$ & $\begin{array}{l}8.66 \\
\pm .05\end{array}$ & 9 & $\begin{array}{l}-.28 \\
\pm .41\end{array}$ & $\begin{array}{c}.11 \\
\pm .29\end{array}$ & $\begin{array}{l}8.65 \\
\pm .06\end{array}$ & $\begin{array}{l}8.60 \\
\pm .06\end{array}$ & 11 & $\begin{array}{r}5.56 \\
\pm .39\end{array}$ & $\begin{array}{c}.52 \\
\pm .24\end{array}$ & $\begin{array}{l}8.43 \\
\pm .05\end{array}$ & $\begin{array}{l}8.66 \\
\pm .04\end{array}$ \\
\hline $\begin{array}{l}\text { No } \\
\text { Flicker }\end{array}$ & & 10 & $\begin{array}{l}-8.4 \\
\pm .29 \\
\end{array}$ & $\begin{array}{l}1.25 \\
\pm .21 \\
\end{array}$ & $\begin{array}{l}9.20 \\
\pm .05\end{array}$ & $\begin{array}{l}8.74 \\
\pm .05 \\
\end{array}$ & 19 & $\begin{array}{c}.64 \\
\pm .25 \\
\end{array}$ & $\begin{array}{l}1.01 \\
\pm .22\end{array}$ & $\begin{array}{l}9.60 \\
\pm .04\end{array}$ & $\begin{array}{l}9.55 \\
\pm .04\end{array}$ & 11 & $\begin{array}{l}9.68 \\
\pm .29\end{array}$ & $\begin{array}{l}1.30 \\
\pm .18\end{array}$ & $\begin{array}{l}8.45 \\
\pm .07\end{array}$ & $\begin{array}{l}8.75 \\
\pm .05\end{array}$ \\
\hline \multirow[t]{2}{*}{$\begin{array}{l}\text { Fast } \\
\text { ON }\end{array}$} & $1 \mathrm{~Hz}$ & 11 & $\begin{array}{c}-8.43 \\
\pm 1.0 \\
4\end{array}$ & $\begin{array}{c}.32 \\
\pm .23\end{array}$ & $\begin{array}{l}9.23 \\
\pm .10\end{array}$ & $\begin{array}{l}8.70 \\
\pm .08\end{array}$ & 11 & $\begin{array}{c}.89 \\
\pm .33\end{array}$ & $\begin{array}{c}.95 \\
\pm .34\end{array}$ & $\begin{array}{l}8.76 \\
\pm .04\end{array}$ & $\begin{array}{l}8.68 \\
\pm .04\end{array}$ & 12 & $\begin{array}{c}5.62 \\
\pm 1.1 \\
7\end{array}$ & $\begin{array}{c}.17 \\
\pm .31\end{array}$ & $\begin{array}{l}8.80 \\
\pm .10\end{array}$ & $\begin{array}{l}8.81 \\
\pm .06\end{array}$ \\
\hline & $4 \mathrm{~Hz}$ & 11 & $\begin{array}{c}-8.70 \\
\pm 1.4 \\
3\end{array}$ & $\begin{array}{c}.10 \\
\pm .27\end{array}$ & $\begin{array}{l}9.13 \\
\pm .10\end{array}$ & $\begin{array}{l}8.73 \\
\pm .06\end{array}$ & 10 & $\begin{array}{c}.60 \\
\pm .58\end{array}$ & $\begin{array}{c}.62 \\
\pm .21\end{array}$ & $\begin{array}{l}8.71 \\
\pm .07\end{array}$ & $\begin{array}{l}8.65 \\
\pm .06\end{array}$ & 12 & $\begin{array}{c}6.38 \\
\pm 1.2 \\
0\end{array}$ & $\begin{array}{c}.75 \\
\pm .22\end{array}$ & $\begin{array}{l}8.50 \\
\pm .04\end{array}$ & $\begin{array}{l}8.70 \\
\pm .06\end{array}$ \\
\hline
\end{tabular}




\section{Table 2 (on next page)}

Summary of within light profile condition and within frequency condition post hoc interactions. 


\begin{tabular}{|c|c|c|c|c|c|}
\hline \multicolumn{3}{|c|}{ Sawtooth Profile } & \multicolumn{3}{|r|}{ Frequency } \\
\hline \multirow[t]{3}{*}{ Fast OFF/Slow ON } & -10D Condition & $1 \mathrm{~Hz}<(4 \mathrm{~Hz}$ No Diff. NF $)$ & $1 \mathrm{~Hz}$ & -10D Condition & Fast OFF $<$ (Fast ON No Diff. NF) \\
\hline & No Lens Condition & $1 \mathrm{~Hz}$ No Diff. NF No Diff. $4 \mathrm{~Hz}$ & & No Lens Condition & Fast OFF No Diff. Fast ON. Fast OFF No Diff. NF \\
\hline & +10D Condition & $1 \mathrm{~Hz}<4 \mathrm{~Hz}<\mathrm{NF}$ & & $+10 \mathrm{D}$ Condition & Fast OFF $<$ (Fast ON No Diff. NF) \\
\hline \multirow[t]{3}{*}{ Fast ON/Slow OFF } & $-10 \mathrm{D}$ Condition & $1 \mathrm{~Hz}$ No Diff. NF No Diff. $4 \mathrm{~Hz}$ & $4 \mathrm{~Hz}$ & $-10 \mathrm{D}$ Condition & Fast OFF No Diff. Fast ON. Fast OFF $<$ NF \\
\hline & No Lens Condition & $1 \mathrm{~Hz}$ No Diff. NF No Diff. $4 \mathrm{~Hz}$ & & No Lens Condition & Fast OFF No Diff. Fast ON. Fast OFF No Diff. NF \\
\hline & $+10 \mathrm{D}$ Condition & $(1 \mathrm{~Hz}$ No Diff. $4 \mathrm{~Hz})<\mathrm{NF}$ & & $+10 \mathrm{D}$ Condition & (Fast OFF No Diff. Fast ON) $<\mathrm{NF}$ \\
\hline
\end{tabular}

1 Note: $<$ denotes more negative refraction (i.e. more myopic). NF $=$ No Flicker. No Diff $=$ No significant difference between conditions. 
Figure 1 (on next page)

Light probe recordings of Fast ON/Slow OFF (Blue) and Fast OFF/Slow ON (Red) sawtooth flicker at a frequency of (a) $1 \mathrm{~Hz}$, and (b) $4 \mathrm{~Hz}$. 


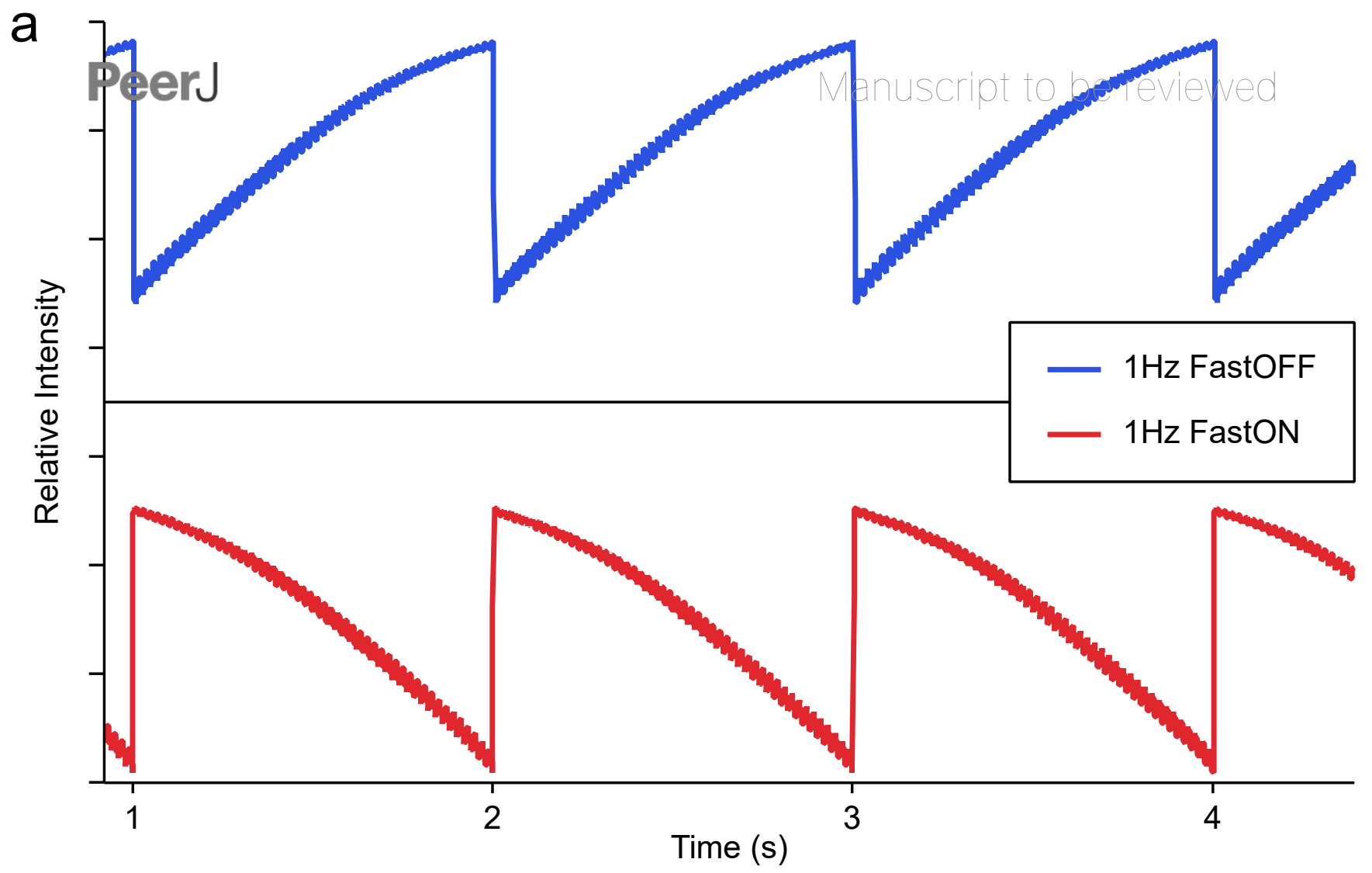

b
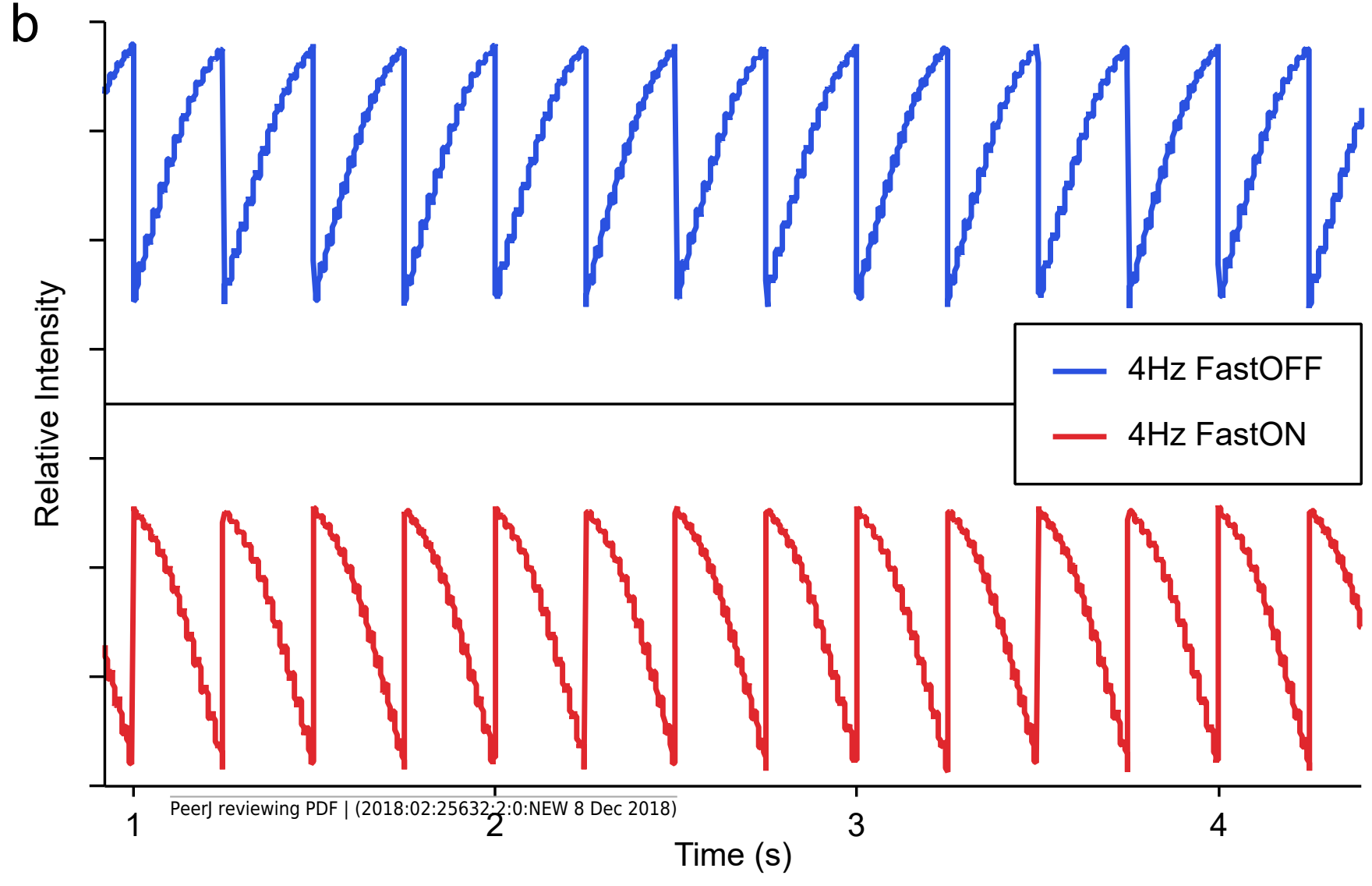
Figure 2

Mean difference ( \pm Standard Error S.E) in biometric measures between experimental and fellow eyes across lens and light conditions.

(a) Refractive difference (diopters), (b) axial length difference ( $\mathrm{mm}),(\mathbf{c})$ vitreous chamber difference $(\mathrm{mm})$, and $(\mathbf{d})$ anterior chamber difference $(\mathrm{mm})$. Asterisks indicate significant differences between condition for post hoc analysis.

a

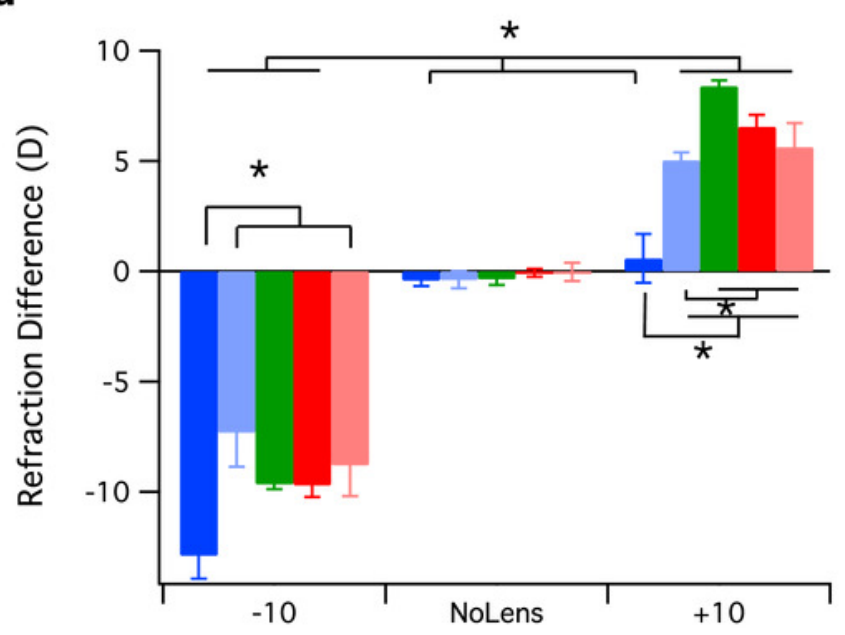

b

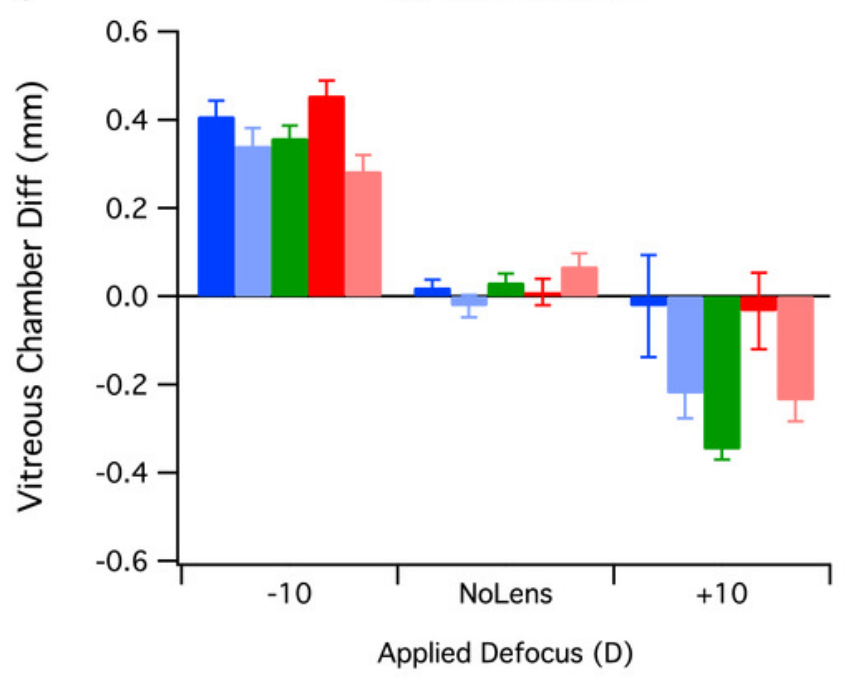

c

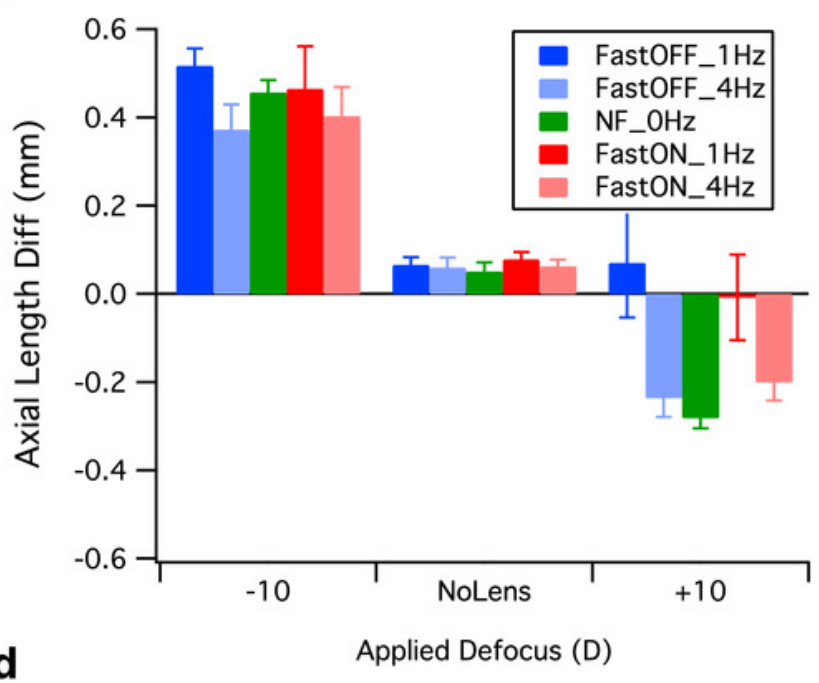

d

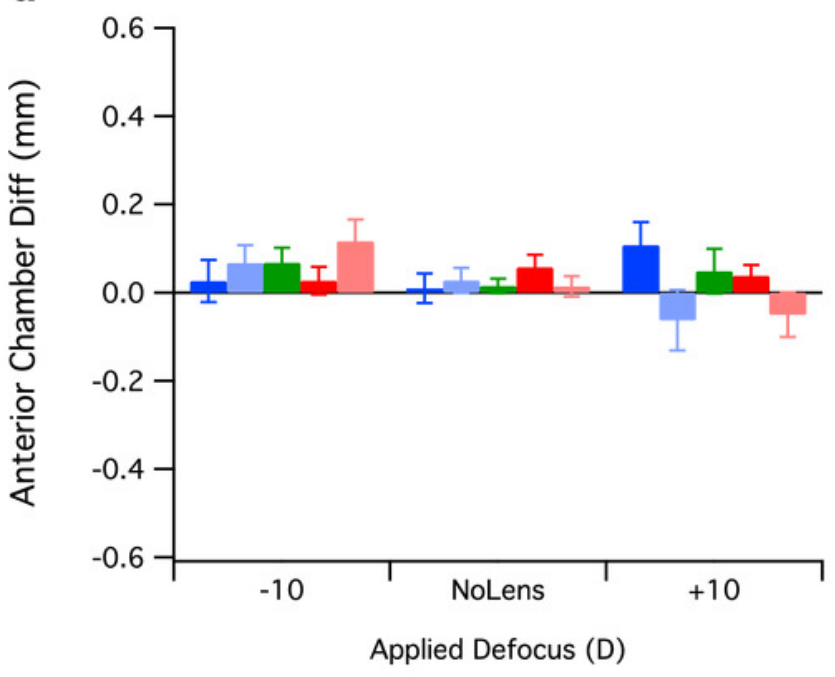

